\title{
PREVENCĈ̣̃O dA XEROSTOMIA E DA MUCOSITE ORAL INDUZIDAS POR RADIOTERAPIA COM USO DO LASER DE BAIXA POTÊNCIA*
}

\author{
Carlos de Oliveira Lopes ${ }^{1}$, Josepa Rigau I Mas², Renato Amaro Zângaro³
}

Resumo OBJETIVO: Verificar se o uso do laser de InGaAIP com comprimento de onda de $685 \mathrm{~nm}$ pode reduzir a incidência de xerostomia, gravidade da mucosite oral e da dor associada à mucosite em pacientes portadores de câncer de cabeça e pescoço submetidos a radioterapia. MATERIAIS E MÉTODOS: Sessenta pacientes portadores de carcinoma de cabeça e pescoço foram submetidos a radioterapia com dose diária de 1,8 a 2,0 Gy e dose final de 45 a $72 \mathrm{~Gy}$. 0 volume salivar foi medido nos dias um, 15, ao final do tratamento e após 15 e 30 dias, e a mucosite oral em avaliações semanais. Vinte e nove pacientes se submeteram a radioterapia sem laser e 31 foram submetidos a radioterapia e laser com dose diária de 2 joules $/ \mathrm{cm}^{2} \mathrm{em}$ pontos pré-determinados da mucosa oral e glândulas parótida e submandibular. RESULTADOS: No grupo submetido a radioterapia e laser, a incidência de mucosite $(p<0,001)$ e dor $(p<0,016)$ foram significativamente menores e o volume salivar se manteve maior $(p<0,001)$ durante e após o tratamento. CONCLUSÃO: Os pacientes submetidos à associação de radioterapia e laser tiveram menor incidência de xerostomia, mucosite oral e dor quando comparados ao grupo de radioterapia sem laser, com resultados com significância estatística.

Unitermos: Laser de baixa potência; Xerostomia; Mucosite; Radioterapia; Quimioterapia.

Abstract Low level laser therapy in the prevention of radiotherapy-induced xerostomia and oral mucositis. OBJECTIVE: To verify if the use of InGaAIP laser with $685 \mathrm{~nm}$ wave length can reduce the xerostomy incidence, the oral mucositis severity and the pain related to mucositis in patients with head and neck cancer submitted to radiotherapy. MATERIALS AND METHODS: Sixty patients presenting head and neck carcinoma were submitted to radiotherapy with daily doses of 1.8 to $2.0 \mathrm{~Gy}$ and a final dose of 45 to $72 \mathrm{~Gy}$. The salivary volume was evaluated in the first and fifteenth days, at the end of the treatment and after 15 and 30 days. The oral mucositis was evaluated on a weekly basis. Twenty-nine patients were submitted to radiotherapy without laser and 31 were submitted to radiotherapy and laser with daily doses of 2 joules/ $\mathrm{cm}^{2}$ in predetermined areas of the oral mucosa and the parotid and submandibular glands. RESULTS: In the group submitted to radiotherapy and laser the incidence of mucositis $(p<0.001)$ and pain $(p<0.016)$ was significantly lower and the salivary volume $(p<0.001)$ was kept higher during and after the treatment. CONCLUSION: The group of patients submitted to radiotherapy and laser had lower incidence of xerostomy, oral mucositis and pain when compared to the group treated with radioteraphy without laser, producing statistically significant results.

Keywords: Low level laser; Xerostomy; Mucositis; Radiotherapy; Chemotherapy.

\section{INTRODUÇÃO}

A radioterapia (RT) em cabeça e pescoço é sempre acompanhada de complicações durante a vigência ou mesmo após o tratamento, principalmente com relação à mucosa oral e glândulas salivares, as quais se manifestam como mucosite e xerosto-

* Trabalho realizado no Instituto de Radioterapia do Vale do Paraíba, São José dos Campos, SP.

1. Médico Radioterapeuta do Instituto de Radioterapia do Vale do Paraíba.

2. Professora Doutora da Unitat Histologia i Neurobiologia, Facultat Medicine i la Ciencies Salut, Universitat Rovira i Virgili, Tarragona, España.

3. Professor do Instituto de Pesquisa e Desenvolvimento da Universidade do Vale do Paraíba.

Endereço para correspondência: Dr. Carlos de Oliveira Lopes. Rua Major Antonio Domingues, 494, Centro. São José dos Campos, SP, 12245-750. E-mail: colopes@uol.com.br

Recebido para publicação em 11/4/2005. Aceito, após revisão, em 5/7/2005. mia, principalmente quando associada com quimioterapia $(\mathrm{QT})^{(\mathbf{1})}$.

A mucosite oral é um problema clínico de difícil controle, podendo comprometer a deglutição, a ingestão de alimentos, a higiene oral e a capacidade de comunicação do paciente, muitas vezes obrigando à interrupção da RT com evidente prejuízo ao tratamento $^{(2-5)}$

A xerostomia se inicia logo nos primeiros dias de RT, sendo mais evidente após doses de $20 \mathrm{~Gy}$. A saliva se torna inicialmente mais espessa e com taxa de eliminação diminuída ${ }^{(6)}$. As alterações do fluxo, volume e viscosidade salivar podem persistir por anos e a recuperação da produção normal dependerá das características de cada paciente e do percentual de glândula salivar irradiada, podendo estar relacio- nadas a danos vasculares dessas glândulas ${ }^{(7)}$. Essa xerostomia pode ser irreversível, com tendência de recuperação de até $50 \%$ da quantia inicial de saliva, caso o volume da parótida irradiado e a dose final de irradiação fique em valores os mais baixos possíveis ${ }^{(\mathbf{8}-\mathbf{1 2})}$.

Várias técnicas são descritas na literatura para prevenção da xerostomia e da mucosite oral, como o uso de amifostina, pilocarpina, Biotene ${ }^{\circledR}$, bochechos com bicarbonato de sódio, uso de antibióticos e anestésicos tópicos, entre outros, todas com suas limitações ${ }^{\mathbf{( 1 3 - 1 9 )}}$.

Lasers de baixa potência (LBP) são lasers que possuem baixa energia, sem potencial fototérmico, sendo utilizados para biomodulação. Os mais usados estão na faixa do vermelho (632 a $780 \mathrm{~nm}$ ), com fótons 
de energia inferior a 2,0 elétron-volt $(\mathrm{eV})$, portanto, inferior à energia da ligação das moléculas biológicas e do DNA, não podendo quebrar ligações químicas e não sendo capazes de induzir mutação e carcinogênese $\mathrm{e}^{\mathbf{( 2 0 - 2 3 )}}$.

O efeito produzido pela irradiação com LBP se baseia na capacidade de modulação de diversos processos metabólicos, mediante a conversão da energia luminosa aportada pelo laser através de processos bioquímicos e fotofísicos, os quais transformam a luz laser em energia útil para a célula $^{(23)}$. O laser visível provoca reações nas mitocôndrias, com incremento na produção de ATP mitocondrial, aumento do consumo de glicose celular, aumento dos teores de cálcio intracelular e do número de mitocôndrias celular.

Aumento no número de mitoses nos ductos de glândulas submandibulares de ratos submetidas à irradiação com laser de GaAs também é descrito ${ }^{(24-30)}$.

Publicações mostram o uso do laser de hélio/neônio como um agente capaz de reduzir a intensidade da mucosite oral e da dor em pacientes submetidos a RT e QT e de pacientes submetidos a transplante autólogo de medula e a RT e QT ${ }^{(31-35)}$.

Trabalhos publicados mostram a eficácia do LBP como um agente estimulador da salivação em pacientes portadores de xerostomia, sialoadenites e síndrome de Sjögren ${ }^{(36-39)}$.

O objetivo deste trabalho é verificar se o uso do laser de InGaAlP com comprimento de onda de $685 \mathrm{~nm}$ pode reduzir a incidência de xerostomia, da gravidade da mucosite oral e da dor associada à mucosite em pacientes portadores de câncer de cabeça e pescoço submetidos à RT.

\section{MATERIAIS E MÉTODOS}

\section{Ética em pesquisa com seres humanos}

Este estudo, obedecendo à resolução $\mathrm{n}^{\mathrm{o}}$ 196/96 do Ministério da Saúde do Brasil, foi aprovado pelo Comitê de Ética em Pesquisa com Seres Humanos da Universidade do Vale do Paraíba, filiado ao Conselho Nacional de Pesquisa com Seres Humanos (Conep), conforme protocolo $\mathrm{n}$ ㅇ L001/ 2002/CEP, sendo que os voluntários assinaram formulário consentido com orientações sobre o projeto.

\section{População e critérios de inclusão e exclusão}

O estudo foi realizado em um grupo de pacientes voluntários, com idade acima de 21 anos, portadores de carcinoma de cabeça e pescoço e não submetidos à RT prévia na região de interesse, e nos quais os campos de irradiação englobassem as glândulas salivares. Os pacientes com prontuário de número ímpar foram denominados "controle", recebendo RT sem laser, e os de prontuário par foram submetidos a RT e laser. Entre os meses de março e setembro de 2002, 60 pacientes foram randomizados em um grupo de 29 pacientes submetidos a RT sem laser e em outro grupo de 31 pacientes submetidos a RT e laser.

Todos os pacientes foram submetidos a cuidados dentários gratuitos iniciais e ao melhor tratamento clínico de suporte durante todo o tratamento.

\section{Aplicação da luz laser}

Foi utilizado laser de InGaAlP com comprimento de onda de $685 \mathrm{~nm}$. A forma de aplicação foi por contato, sendo três pontos em cada parótida, um submandibular direito e esquerdo, dois pontos em mucosa jugal direita e esquerda, um ponto em cada lado do soalho de boca, dois pontos em língua móvel, um ponto em pilar direito, outro em pilar esquerdo e outro na região da úvula, todos com densidade de energia de 2,0 joules $/ \mathrm{cm}^{2}$ por ponto.

Os parâmetros do laser foram potência nominal de $50 \mathrm{~mW}$ e real de $35 \mathrm{~mW}$ em uma fibra com $400 \mu \mathrm{m}$ de diâmetro, tempo de irradiação de $58 \mathrm{~s}$, comprimento de onda de $685 \mathrm{~nm}$ e densidade de dose de 2,0 joules $/ \mathrm{cm}^{2}$. Caso o ponto de aplicação do laser estivesse a menos de $1 \mathrm{~cm}$ da área tumoral, a aplicação do LBP não era realizada naquele local ${ }^{(33)}$.

\section{Radioterapia}

Foi realizada diariamente, com o uso de acelerador linear Varian ${ }^{\circledR}$ (Varian Medical Systems), com fótons com $4 \mathrm{MeV}$ de energia. As áreas irradiadas foram delimitadas conforme princípios técnicos constantes

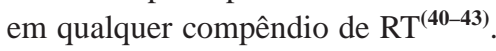

Os pacientes foram submetidos a exames rotineiros de estadiamento, como exames clínico e endoscópico, laboratoriais, tomografias, biópsias, etc.
Os pacientes eram posicionados deitados em decúbito dorsal, imobilizados por máscara termoplástica e submetidos a radiografias de controle.

A dose diária variou entre 1,8 e 2,0 Gy, calculada no meio do plano, e a dose final ficou entre 45 e 72 Gy, em 25 a 40 dias de tratamento. Os campos usados foram o cérvico-facial direito e esquerdo e o das fossas supraclaviculares.

\section{Quimioterapia}

A QT utilizada em associação com a RT foi cisplatina em esquema de uma dose semanal de $40 \mathrm{mg} / \mathrm{m}^{2^{(44)}}$.

\section{Avaliação da xerostomia}

Foi realizada conforme esquema proposto pelo Radiation Therapy Oncology Group (RTOG) 97-09 para sialometria ${ }^{(45)}$.

A avaliação inicial foi realizada no primeiro dia do tratamento de RT e serviu como um indicador do nível basal de produção salivar. A produção não estimulada de saliva foi avaliada antes da estimulada. Inicialmente, o paciente foi orientado para deglutir toda a saliva que estivesse depositada em sua boca. A seguir, foi orientado para acumular saliva, sem degluti-la, por um período de 60 segundos. Esta saliva foi acumulada em um frasco graduado de 50 $\mathrm{ml}$. Este processo foi repetido mais quatro vezes, totalizando cinco tomadas em cinco minutos.

A estimulação de produção de saliva foi feita com a utilização de solução de citrato de sódio a $2 \%$ aplicada nas bordas dorsolaterais da língua, com o uso de cotonete, por cinco vezes em um período de dois minutos (aos 0, 30, 60, 90 e 120 segundos). Antes da aplicação da solução, a boca era limpa de qualquer secreção e, posteriormente, era novamente limpa com a retirada da solução de citrato residual. A saliva foi então coletada do mesmo modo como o foi para o procedimento de salivação não estimulada. Essas medições foram feitas nos dias 1,15 , ao final do tratamento e em 15 e 30 dias pós-tratamento.

\section{Avaliação da mucosite oral}

Foi realizada uma vez por semana, sendo o grau de mucosite classificado conforme preconizado pelo National Cancer Institute (NCI), como mostra a Tabela $1^{(46)}$. 


\section{Avaliação da dor}

A avaliação foi feita conforme escala numérica analógica, em que o paciente era orientado a dar uma nota para o seu grau de dor em mucosa oral (Figura 1).

\section{Análise estatística}

Constou das comparações entre os dois grupos e incluiu cálculo de medidas de tendência central (média) e dispersão (desviopadrão) para variáveis quantitativas, e distribuições de freqüências (\%) para as variáveis qualitativas. Foi utilizado teste de associação do qui-quadrado para verificar a associação entre variáveis qualitativas. Nas tabelas $2 \times 2$, quando pelo menos uma das freqüências esperadas foi menor do que 5, o teste exato de Fisher foi utilizado. Para a comparação de medidas de variáveis quantitativas (idade e doses de radiação) entre os grupos de estudo foi utilizado o teste $t$ de Student para amostras independentes. Quando a suposição da distribuição normal da variável não foi alcançada, foi utilizado o teste de Mann-Whitney (não paramétrico). $\mathrm{Na}$ análise de variância de medidas repetivas foi utilizada a ANOVA, sendo o tempo a medida repetitiva e o grupo de estudo a variável independente. Quando as variâncias entre os grupos não eram homogêneas, utilizou-se o teste de Friedman, que é o equivalente não paramétrico. Na presença de diferenças estatisticamente significativas a ANOVA foi complementada pelo teste de múltiplas comparações de Tukey-HSD.

\section{RESULTADOS}

Foram analisados os resultados de 60 pacientes, sendo que 29 foram submetidos a RT sem laser e 31 foram tratados com RT e laser. A maioria dos pacientes era do sexo masculino (83,3\%). A idade variou de 28 a 88 anos (média de 57,4 \pm 13,9 anos).

As localizações mais freqüentes dos tumores primários foram laringe $(18,3 \%)$, amígdala $(10,0 \%)$ e língua $(8,3 \%)$. O tipo histológico predominante foi o carcinoma espinocelular $(85,0 \%)$.

A maioria dos pacientes apresentava tumores avançados (estádio clínico IV) $(76,7 \%)$. Trinta e quatro pacientes $(56,7 \%)$ não foram submetidos a cirurgia e 31 não receberam QT $(51,7 \%)$ (Tabela 2$)$.

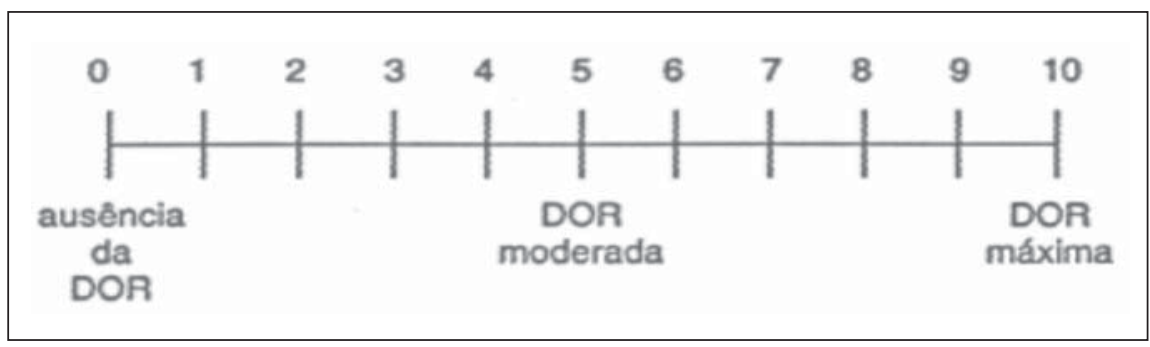

Figura 1. Escala analógica visual para avaliação da dor.

Tabela 1 Classificação de graus de mucosite $-\mathrm{NCl}$.

\begin{tabular}{|c|l|}
\hline Grau de mucosite & \\
\hline 0 & Nenhum \\
1 & Eritema da mucosa \\
2 & Placas pseudomembranosas menores que 1,5 cm de diâmetro e não contíguas \\
3 & $\begin{array}{l}\text { Pseudomembranosas confluentes, em geral placas contíguas maiores que 1,5 cm } \\
\text { de diâmetro } \\
4\end{array}$ \\
& Necrose e/ou ulceração profunda ou sangramento não induzido por trauma \\
\hline
\end{tabular}

Tabela 2 Número e percentual de pacientes segundo variáveis demográficas, tipo de tumor, cirurgia e/ou quimioterapia e grupo estudo (sem laser $\times$ com laser).

\begin{tabular}{|c|c|c|c|}
\hline Variável & Categoria & Sem laser & Com laser \\
\hline \multirow[t]{2}{*}{ Sexo } & Masculino & $25(86,2 \%)$ & $25(80,6 \%)$ \\
\hline & Feminino & $4(13,8 \%)$ & $6(19,4 \%)$ \\
\hline \multirow[t]{12}{*}{ Localização do tumor } & Lábio inferior & - & $1(3,2 \%)$ \\
\hline & Língua & $5(17,2 \%)$ & - \\
\hline & Assoalho boca/gengiva & $1(3,4 \%)$ & $1(3,2 \%)$ \\
\hline & Palato duro e mole & $3(10,3 \%)$ & $2(6,5 \%)$ \\
\hline & Mucosa jugal/retromolar & $2(6,9 \%)$ & $4(12,9 \%)$ \\
\hline & Glândulas salivares & $2(6,9 \%)$ & $4(12,9 \%)$ \\
\hline & Amígdala/orofaringe & $4(13,8 \%)$ & $4(12,9 \%)$ \\
\hline & Rinofaringe & $2(6,9 \%)$ & $1(3,2 \%)$ \\
\hline & Seio piriforme/hipofaringe & $4(13,8 \%)$ & $2(6,5 \%)$ \\
\hline & Seio maxilar & $1(3,4 \%)$ & $3(9,7 \%)$ \\
\hline & Laringe & $4(13,8 \%)$ & $7(22,6 \%)$ \\
\hline & Conduto auditivo & $1(3,4 \%)$ & $2(6,5 \%)$ \\
\hline \multirow[t]{4}{*}{ Estádio clínico TNM } & I & - & $2(6,5 \%)$ \\
\hline & II & $1(3,4 \%)$ & $1(3,2 \%)$ \\
\hline & III & $3(10,3 \%)$ & $6(19,4 \%)$ \\
\hline & IV & $25(86,2 \%)$ & $22(70,9 \%)$ \\
\hline \multirow[t]{6}{*}{ Tipo histológico } & Carcinoma espinocelular & $27(93,1 \%)$ & $24(77,4 \%)$ \\
\hline & Adenocarcinoma & $1(3,4 \%)$ & $2(6,5 \%)$ \\
\hline & Carcinoma basocelular & - & $1(3,2 \%)$ \\
\hline & mucoepidermóide & $1(3,4 \%)$ & $1(3,2 \%)$ \\
\hline & Adenoma/tumor misto & - & $2(6,5 \%)$ \\
\hline & Carcinoma indiferenciado & - & $1(3,2 \%)$ \\
\hline \multirow[t]{2}{*}{ Cirurgia } & Não & 17 (58,6\%) & $17(54,8 \%)$ \\
\hline & Sim & $12(41,4 \%)$ & $14(45,2 \%)$ \\
\hline \multirow[t]{2}{*}{ Quimioterapia } & Não & $14(48,3 \%)$ & $17(54,8 \%)$ \\
\hline & Sim & $15(51,7 \%)$ & $14(45,2 \%)$ \\
\hline
\end{tabular}


Doses em glândulas salivares: Não foram observadas diferenças estatisticamente significativas entre as médias da dose total nas glândulas parótida direita $(p$ $=0,081)$ e esquerda $(p=0,090)$, submandibular direita $(p=0,081)$ e esquerda $(p=$ $0,090)$, sublingual direita $(p=0,081) \mathrm{e}$ esquerda $(p=0,090)$ nos grupos tratados com ou sem laser (Tabela 3).

Fluxo salivar estimulado: Foi observada diferença estatisticamente significativa entre os grupos $(p<0,001)$ (Tabela 4).

Fluxo salivar não estimulado: Houve diferença estatisticamente significativa entre os grupos $(p<0,001)$ (Tabela 5).

Mucosite - escore da NCI: Foram observadas diferenças estatisticamente significativas entre os momentos $(p<0,001) \mathrm{e}$ entre os grupos $(p<0,001)$. Foi observada interação estatisticamente significativa entre momento e grupo ( $p<0,001)$, isto é, a variação entre os momentos é diferente nos dois grupos de estudo (Tabela 6).

Dor - escala analógica: Houve diferença estatisticamente significativa entre os grupos $(p<0,001)$ (Tabela 7$)$.

\section{DISCUSSÃO}

Análise univariada dos dados avaliados mostrou não haver diferença estatisticamente significativa entre os grupos quanto aos quesitos sexo, idade, enfermidade de base, estadiamento, doses de irradiação nas glândulas salivares, associação com cirurgia ou quimioterapia, tipo histológico e localização do tumor primário.

As avaliações dos dados referentes à incidência de mucosite oral, dor e xerostomia mostraram que os pacientes submetidos a RT associada a laser tiveram índices significativamente inferiores destes eventos em comparação ao grupo de RT sem laser. Bensadoun et al. ${ }^{(\mathbf{3 3})}$ fizeram estudo randomizado com 30 pacientes com uso do laser de $\mathrm{He} / \mathrm{Ne}$, mostrando bons resultados na diminuição da gravidade da dor e da mucosite radioinduzida em pacientes submetidos a RT em cabeça e pescoço. Em nosso trabalho incluímos não só um maior número de casos — um total de 60 como também igualamos as condições de higiene oral inicial de todos eles e o melhor cuidado dentário de suporte durante o tratamento.

Tabela 3 Médias e desvios-padrão da dose total de radiação (Gy) nas glândulas parótida direita e esquerda, submandibular direita e esquerda, sublingual direita e esquerda (sem laser $\times$ com laser).

\begin{tabular}{|l|c|c|c|}
\hline Variável & Sem laser & Com laser & $p^{*}$ \\
\hline Parótida direita & $61,5(13,2)$ & $57,2(14,1)$ & 0,081 \\
Parótida esquerda & $61,5(13,2)$ & $57,2(14,5)$ & 0,090 \\
Submandibular direita & $61,5(13,2)$ & $57,2(14,1)$ & 0,081 \\
Submandibular esquerda & $61,5(13,2)$ & $57,2(14,5)$ & 0,090 \\
Sublingual direita & $61,5(13,2)$ & $57,2(14,1)$ & 0,081 \\
Sublingual esquerda & $61,5(13,2)$ & $57,2(14,5)$ & 0,090 \\
\hline
\end{tabular}

* Nível de significância estatística segundo o teste de Mann-Whitney.

Tabela 4 Médias e desvios-padrão do fluxo salivar estimulado $(\mathrm{ml})$ na avaliação inicial, ao término e na segunda avaliação após o término do tratamento, segundo o grupo de estudo (sem laser $\times$ com laser).

\begin{tabular}{|l|l|l|l|}
\hline Grupo de estudo & \multicolumn{1}{|c|}{ Sem laser } & Com laser & \multicolumn{1}{|c|}{$p^{*}$} \\
\hline Início & $4,2(1,9)$ & $4,5(2,3)$ & $\mathrm{SL}=\mathrm{CL}(p=0,978)$ \\
Término & $1,5(0,6)$ & $4,1(1,8)$ & $\mathrm{SL} \neq \mathrm{CL}(p<0,001)$ \\
Segundo pós-término & $1,7(1,9)$ & $4,6(1,8)$ & $\mathrm{SL} \neq \mathrm{CL}(p<0,001)$ \\
Variação entre & $\mathrm{I} \neq \mathrm{T}(p<0,001)$ & $\mathrm{I}=\mathrm{T}(p=0,869)$ & \\
os momentos $\left(p^{*}\right)$ & $\mathrm{I} \neq \mathrm{PT}(p<0,001)$ & $\mathrm{I}=\mathrm{PT}(p=0,999)$ & \\
& $\mathrm{T}=\mathrm{PT}(p=0,999)$ & $\mathrm{T}=\mathrm{PT}(p=0,774)$ & \\
\hline
\end{tabular}

* Nível de significância estatística segundo o teste de múltiplas comparações de Tukey-HSD. I, início; T, término; PT, pós-término; SL, sem laser; CL, com laser.

Tabela 5 Médias e desvios-padrão do fluxo salivar não estimulado ( $\mathrm{ml}$ ) na avaliação inicial, ao término e na segunda avaliação após o término do tratamento, segundo o grupo de estudo (sem laser $\times$ com laser).

\begin{tabular}{|l|l|l|l|}
\hline Grupo de estudo & \multicolumn{1}{|c|}{ Sem laser } & \multicolumn{1}{|c|}{ Com laser } & \multicolumn{1}{c|}{$p^{*}$} \\
\hline Início & $3,5(1,7)$ & $3,8(2,1)$ & $\mathrm{SL}=\mathrm{CL}(p=0,720)$ \\
Término & $1,4(0,5)$ & $3,8(1,7)$ & $\mathrm{SL} \neq \mathrm{CL}(p<0,001)$ \\
Segundo pós-término & $1,2(0,3)$ & $4,1(1,8)$ & $\mathrm{SL} \neq \mathrm{CL}(p<0,001)$ \\
Variação entre & $\mathrm{I} \neq \mathrm{T}(p<0,001)$ & $\mathrm{I}=\mathrm{T}(p=0,998)$ & \\
os momentos $\left(p^{*}\right)$ & $\mathrm{I} \neq \mathrm{PT}(p<0,001)$ & $\mathrm{I}=\mathrm{PT}(p=0,878)$ & \\
& $\mathrm{T}=\mathrm{PT}(p=0,985)$ & $\mathrm{T}=\mathrm{PT}(p=0,652)$ & \\
\hline
\end{tabular}

* Nível de significância estatística segundo o teste de múltiplas comparações de Tukey-HSD. I, início; T, término; PT, pós-término; SL, sem laser; CL, com laser.

Tabela 6 Médias e desvios-padrão do escore de mucosite ( $\mathrm{NCl})$ na avaliação inicial, após quatro semanas e ao término do tratamento, segundo o grupo de estudo (sem laser $\times$ com laser).

\begin{tabular}{|l|l|l|l|}
\hline Grupo de estudo & \multicolumn{1}{|c|}{ Sem laser } & \multicolumn{1}{|c|}{ Com laser } & \multicolumn{1}{c|}{$p^{*}$} \\
\hline Início & $0,4(0,5)$ & $0,3(0,5)$ & $\mathrm{SL}=\mathrm{CL}(p=1,000)$ \\
Após quatro semanas & $1,8(1,0)$ & $0,7(0,9)$ & $\mathrm{SL} \neq \mathrm{CL}(p<0,001)$ \\
Término & $1,8(1,1)$ & $0,8(0,9)$ & $\mathrm{SL} \neq \mathrm{CL}(p<0,001)$ \\
Variação entre & $\mathrm{I} \neq 4 \mathrm{~s}(p<0,001)$ & $\mathrm{I}=4 \mathrm{~s}(p=0,419)$ & \\
os momentos $\left(p^{*}\right)$ & $\mathrm{I} \neq \mathrm{T}(p<0,001)$ & $\mathrm{I}=\mathrm{T}(p=0,221)$ & \\
& $4 \mathrm{~S}=\mathrm{T}(p=1,000)$ & $4 \mathrm{~S}=\mathrm{T}(p=0,999)$ & \\
\hline
\end{tabular}

* Nível de significância estatística segundo o teste de múltiplas comparações de Tukey-HSD. I, início; 4s, quatro semanas; T, término; SL, sem laser; CL, com laser.

Os pacientes submetidos ao tratamento RT e laser evoluíram com menor incidência de mucosite e dor devido ao tratamento, estando nossos dados de acordo com Cowen et al. ${ }^{(32)}$, que compararam a evolução da mucosite oral em 30 pacientes que receberam transplante autólogo de medula e RT durante o período de 1993 a 1995, mostrando redução significativa da mucosite e considerando a aplicação do 
Tabela 7 Médias e desvios-padrão do escore de dor na avaliação inicial, após quatro semanas e ao término do tratamento, segundo o grupo de estudo (sem laser $\times$ com laser).

\begin{tabular}{|l|l|l|l|}
\hline Grupo de estudo & \multicolumn{1}{|c|}{ Sem laser } & \multicolumn{1}{|c|}{ Com laser } & \multicolumn{1}{c|}{$p^{*}$} \\
\hline Início & $0,0(0,0)$ & $0,1(0,6)$ & $\mathrm{SL}=\mathrm{CL}(p=0,999)$ \\
Após quatro semanas & $2,2(2,6)$ & $0,9(1,9)$ & $\mathrm{SL} \neq \mathrm{CL}(p<0,016)$ \\
Término & $2,4(2,5)$ & $0,7(1,5)$ & $\mathrm{SL} \neq \mathrm{CL}(p<0,001)$ \\
Variação entre & $\mathrm{I} \neq 4 \mathrm{~s}(p<0,001)$ & $\mathrm{I}=4 \mathrm{~s}(p=0,348)$ & \\
os momentos $\left(p^{\star}\right)$ & $\mathrm{I} \neq \mathrm{T}(p<0,001)$ & $\mathrm{I}=\mathrm{T}(p=0,692)$ & \\
& $4 \mathrm{~s}=\mathrm{T}(p=0,996)$ & $4 \mathrm{~s}=\mathrm{T}(p=0,994)$ & \\
\hline
\end{tabular}

* Nível de significância estatística segundo o teste de múltiplas comparações de Tukey-HSD. I, início; 4s, quatro semanas; T, término; SL, sem laser; CL, com laser.

laser de forma profilática como tendo melhores resultados que a de forma curativa para mucosite já instalada.

Em nosso trabalho a aplicação foi realizada desde o início da RT, prolongandose durante todo o tratamento.

Por motivos sociais ou de ordem pessoal, alguns pacientes não seguiram esta rigidez de conduta, tendo faltado ao tratamento com laser, às vezes por até cinco dias consecutivos. Como exemplo, temos um paciente portador de carcinoma de rinofaringe realizando $\mathrm{RT}+\mathrm{QT}+$ laser que decidiu não comparecer às sessões de laser por uma semana devido ao excesso de saliva. $\mathrm{O}$ agravamento da mucosite o trouxe de volta ao plano terapêutico inicial, havendo redução da mucosite logo após alguns dias de aplicação de laser. Isto nos levou a pensar que a aplicação diária de laser talvez não fosse necessária, sendo que planejamos no futuro estudar um grupo com três sessões semanais e compararmos o resultado.

Observamos, durante a realização do trabalho, que no grupo de RT e laser todos os pacientes completaram o tratamento previsto, enquanto no grupo de RT sem laser quatro pacientes de um total de 29 $(13,8 \%)$ tiveram os seus tratamentos suspensos devido a mucosite. Esta observação está de acordo com o trabalho de Balakirev et al. ${ }^{(35)}$ sobre o uso do laser para a prevenção de mucosite oral, no qual se concluiu que o tempo de parada de tratamento necessário para manejo das complicações devidas às injúrias da RT e QT em pacientes pediátricos era reduzido em 1,5 vez até 2 vezes no grupo de submetido associado a laser. Não encontramos, na literatura, relato de estudos realizados no sentido de mostrar o valor do uso do laser para prevenção da xerostomia durante a vigência de RT. Existem vários trabalhos mostrando o valor do laser como estimulador de glândulas salivares. Entre eles, citamos Kats ${ }^{(38,39)}$ com o uso do laser de hélio/neônio em sialoadenites, Fructuoso e Moset ${ }^{(\mathbf{3 6})}$ e Takeda ${ }^{(24)}$ usando laser de GaAs com comprimento de onda de $904 \mathrm{~nm}$, e Nagasawa $^{(37)}$ usando laser de GaAlAs com comprimento de onda de $820 \mathrm{~nm}$ em pacientes portadores da síndrome de Sjögren, com resultados que mostraram melhora do fluxo de saliva produzida pela parótida após a estimulação pela luz laser.

Nossos dados estão de acordo com a literatura e mostraram que no grupo de pacientes submetidos à terapia associada de RT e laser houve menor queda do volume salivar do que no grupo submetido à RT sem laser, em que todos os pacientes mostraram queda progressiva dos volumes de saliva estimulada e não estimulada, com março de 2004. uma tendência para volumes menores que 1,0 ml. No grupo submetido ao tratamento de RT e laser, em alguns casos ocorreu aumento do volume salivar durante a RT, fato que nunca havíamos observado durante todos os anos de trabalho na especialidade, mesmo utilizando bochechos com bicarbonato de sódio, Biotene ${ }^{\circledR}$, saliva artificial e outros tratamento clínicos de suporte de rotina da prática médica.

As reavaliações que temos feito, embora sem valor estatístico, mostram que o volume salivar dos pacientes submetidos a RT e laser tem-se mantido em níveis superiores aos dos pacientes submetidos à RT sem laser (Figura 2).

É nossa primeira impressão que o ganho tende a ser prolongado, sendo que os pacientes deverão ser acompanhados durante os próximos meses e anos, e reavaliações sucessivas de seu volume salivar servirão para mostrar se a manutenção deste benefício inicial persistirá.

\section{CONCLUSÕES}

A incidência de xerostomia foi significativamente menor nos pacientes submetidos a RT e laser.

A utilização do laser em associação com RT diminuiu a gravidade da mucosite oral.

A dor associada à mucosite oral também é significativamente reduzida nos pacientes submetidos a RT e laser.

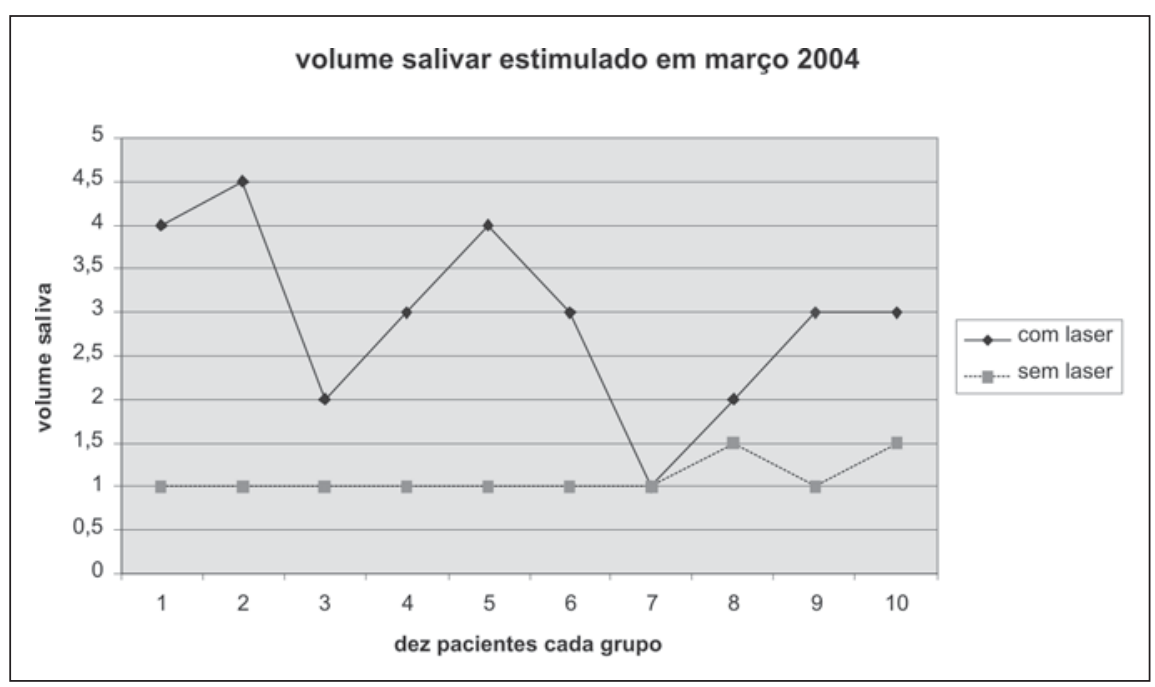

Figura 2. Volume salivar medido em 20 pacientes que responderam ao convite para nova avaliação em 


\section{REFERÊNCIAS}

1. Trotti A. Toxicity in head and neck cancer: a review of trends and issues. Int J Radiat Oncol Biol Phys 2000;47:1-12.

2. Bonassa EMA. Enfermagem em quimioterapia. São Paulo: Atheneu, 1998;238.

3. Fontelonga A. Mucosite. A saúde na internet. São Paulo; 2001 [acessado em 5 nov 2001]. Disponível em: http://www.saudenainternet-rede MNI.htm

4. Carl W, Havens J. The cancer patient with severe mucositis. Curr Rev Pain 2000;3:197-202.

5. Devita VT Jr. Oral complications. In: Cancer: principles and practice of oncology. 6th ed. Philadelphia, PA: Lippincott Williams \& Wilkins, 2001; 2881-2888.

6. Semba SE, Mealey BL, Hallmon WW. The head and neck radiotherapy patient: Part 1 - oral manifestations of radiation therapy. Compend Contin Educ Dent 1994;15:252-260.

7. Fajardo LF, Bertrong M, Anderson RE. Salivary glands In: Fajardo LF, Bertrong M, Anderson RE, editors. Radiation pathology. Minnesota: Oxford University Press, 2001;265-270.

8. Chao KS, Deasy JO, Markmon J, et al. A prospective study of salivary function sparing in patients with head-and-neck cancers receiving intensitymodulated or three-dimensional radiation therapy: initial results. Int J Radiat Oncol Biol Phys 2001; 49:907-916.

9. Burlage FR, Coppes RP, Meertens H, et al. Parotid and submandibular/sublingual salivary flow during high dose radiotherapy. Radiother Oncol 2001;61: 271-274.

10. Roesink JM, Moerland MA, Battermann JJ, et al. Quantitative dose-volume response analysis of changes in parotid gland function after radiotherapy in the head-and-neck region. Int J Radiat Oncol Biol Phys 2001;51:938-946.

11. Maes A, Weltens C, Flamen P, et al. Preservation of parotid function with uncomplicated conformal radiotherapy. Radiother Oncol 2002;63:203-211.

12. Cheng VST, Downs J, Herbert D, Aramany M. The function of the parotid gland following radiation therapy for head and neck cancer. Int J Radiat Oncol Biol Phys 1981;7:253-258.

13. Johnson JT, Ferreti GA, Nethery WJ, et al. Oral pilocarpine for post-irradiation xerostomia in patients with head and neck cancer. N Engl J Med 1993;329:390-395.

14. Rieke JW, Hafermann MD, Johnson JT, et al. Oral pilocarpine for radiation-induced xerostomia: integrated efficacy and safety results from two prospective randomized clinical trials. Int J Radiat Oncol Biol Phys 1995;31:661-669.

15. Schuchter LM, Hensley ML, Meropal NJ, et al. 2002 Update of recommendations for the use of chemotherapy and radiotherapy protectants: clini- cal practice guidelines of the American Society of Clinical Oncology. J Clin Oncol 2002;20:28952903.

16. Warde P, Krall B, O'Sullivan B, et al. A phase II study of Biotene in the treatment of postradiation xerostomia in patients with head and neck cancer. Support Care Cancer 2000;8:203-208.

17. Antonadou D, Pepelassi M, Synodinou M, Puglisi M, Throuvalas N. Prophylactic use of amifostine to prevent radiochemotherapy-induced mucositis and xerostomia in head-and-neck cancer. Int J Radiat Oncol Biol Phys 2002;52:739-747.

18. Epstein JB, Schubert MM. Synergistic effect of sialagogues in management of xerostomia after radiation therapy. Oral Surg Oral Med Oral Pathol 1987;64:179-182.

19. Schubert MM, Franquini JC, Niccoli-Filho F, et al. Effects of low-energy laser on oral mucositis: a phase I/II pilot study. Cancer Res Weekly 1994;7:14.

20. Brugnera Jr A, Pinheiro ALB. Lasers na odontologia moderna. São Paulo, SP: Pancast, 1998.

21. Tunér J, Hode L. Low level laser therapy. Graengesberg, Sweden: Prima Books, 1999;403.

22. Maldonado EP. Mecanismos de interação laser-tecido [apostila do curso de mestrado profissionalizante de lasers em odontologia do Ipen]. São Paulo, SP: Ipen, 2000.

23. Cisneros JLV. Láser y fuentes de luz pulsada intensa em dermatología y dermocosmética. Madrid: Aula Médica Ediciones, 2000.

24. Takeda Y. Irradiation effect of low-energy laser on rat submandibular salivary gland. J Oral Pathol 1988;17:91-94.

25. Bortolleto R. Efeito da radiação do laser de baixa potência no potencial de membrana de mitocôndrias em células in-vitro. (Dissertação de Mestrado). São José dos Campos, SP: Universidade do Vale do Paraíba, 2000.

26. Rigau J. Acción de la luz láser a baja densidad en la modulación de la función celular. (Tesis de Doctorado). Reus-Tarragona, Espanha: Universidad Rovira y Virgili, 1996.

27. Karu TI. Photobiological fundamentals of lowpower laser therapy. J Quantum Electronics Moscow 1987;QE-23(10):1703-1717.

28. Mester AF, Mester A. Wound healing. Laser Therapy 1989;1:7-15.

29. Hashieh IA, Tardieu C, Franquin JC. Helium-neon laser irradiation is not a stressful treatment: a study on heat-shock protein (HSP70) level. Lasers Surg Med 1997;4 :451-460.

30. Genovese WJ. Laser de baixa intensidade: aplicações terapêuticas em odontologia. São Paulo, SP: Lovise, 2000.

31. Barasch A, Peterson DE, Tanzer JM, et al. Heliumneon laser effects on conditioning induced oral mucositis in bone marrow transplantation patients. Cancer 1995;76:2550-2556.
32. Cowen D, Tardieu C, Schubert M, et al. Low energy helium-neon laser in the prevention of oral mucositis in patients undergoing bone marrow transplant: results of a double blind randomized trial. Int J Radiat Oncol Biol Phys 1997;38:697-703.

33. Bensadoun RJ, Franquin JC, Ciais G. Low-energy $\mathrm{He} / \mathrm{Ne}$ laser in the prevention of radiation-induced mucositis. A multicenter phase III randomized study in patients with head and neck cancer. Support Care Cancer 1999;7:244-252.

34. Plevová P. Prevention and treatment of chemotherapy and radiotherapy induced oral mucositis: a review. Oral Oncol 1999;5:453-470.

35. Balakirev SA, Gusev LI, Kasanova MB, et al. Lowintensity laser therapy in pediatric oncology. Vopr Onkol 2000;46:459-461.

36. Fructuoso FJG, Moset J M. Randomized double blind study on biostimulatory effect of laser irradiation of the parotid gland in patients suffering of Sjögren syndrome. Invest y Clinica Laser 1987; 1 : 18-25.

37. Nagasawa A. Application of LLLT in dentistry. In: Ohshiro T, editor. Low-reactive laser therapy. Practical applications. London: John Wiley \& Sons, 1991;76.

38. Kats AG. Low-energy laser radiation in the combined treatment of salivary gland inflammation. Stomatologia (Mosk) 1993;4:32-36.

39. Kats AG, Belastotskaia IM, Malamud ZP, et al. Remote results of the complex treatment of chronic sialadenitis using the helium-neon laser. Vestnik Khirurgii Imeni I-I Grekova 1985;135:39-42.

40. Hall EJ. Radiobiology for the radiologist. 4th ed Philadelphia, PA: JB Lippincott, 1994;1-13.

41. Bentel GC. Radiation therapy planning. 2nd ed. New York, NY: McGraw-Hill, 1996;268-330.

42. Perez CA, Brady LW. Principles and practice of radiation oncology. 3rd ed. Philadelphia, PA: Lippincott-Raven, 1998;981-1002.

43. Leibel SA, Phillips TL. Textbook of radiation oncology. Philadelphia, PA: WB Saunders, 1998; 412-526.

44. Gomes R. Oncologia básica. Rio de Janeiro, RJ: Revinter, 1997

45. Radiation Therapy Oncology Group. A phase III study to test the efficacy of the prophylatic use of oral pilocarpine to reduce hyposalivation and mucositis associated with curative radiation therapy in head and neck cancer patients. RTOG97-09. Philadelphia, PA: Radiation Therapy Oncology Group, 1999.

46. Trotti A, Byhardt R, Stetz J, et al. Common toxicity criteria: version 2.0. An improved reference for grading the effects of cancer treatment: impact on radiotherapy. Int J Radiat Biol Oncol Phys 2000; 47:13-47. 